\title{
Biomimetic Three-Dimensional Cultures Significantly Increase Hematopoietic Differentiation Efficacy of Embryonic Stem Cells
}

\author{
HUI LIU, Ph.D., and KRISHNENDU ROY, Ph.D.
}

\begin{abstract}
Stem cell-based tissue engineering is a promising technology in the effort to create functional tissues of choice. To establish an efficient approach for generating hematopoietic cell lineages directly from embryonic stem (ES) cells and to study the effects of three-dimensional (3D) biomaterials on ES cell differentiation, we cultured mouse ES cells on 3D, highly porous, biomimetic scaffolds. Cell differentiation was evaluated by microscopy and flow cytometry analysis with a variety of hematopoiesis-specific markers. Our data indicate that ES cells differentiated on porous 3D scaffold structures developed embryoid bodies (EBs) similar to those in traditional two-dimensional (2D) cultures; however, unlike 2D differentiation, these EBs integrated with the scaffold and appeared embedded in a network of extracellular matrix. Most significantly, the efficiency of hematopoietic precursor cell (HPC) generation on 3D, as indicated by the expression of various HPC-specific surface markers (CD34, Sca-1, Flk-1, and c-Kit) and colony-forming cell (CFC) assays, was reproducibly increased (about 2-fold) over their 2D counterparts. Comparison of static and dynamic 3D cultures demonstrated that spinner flask technology also contributed to the higher hematopoietic differentiation efficiency of ES cells seeded on scaffolds. Continued differentiation of 3D-derived HPCs into the myeloid lineage demonstrated increased efficiency (2-fold) of generating myeloid compared with differentiation from 2D-derived HPCs.
\end{abstract}

\section{INTRODUCTION}

$\mathbf{T}$ TREMENDOUS PROGRESs has been made in differentiating embryonic stem (ES) cells into a variety of somatic lineages. Several research groups have demonstrated the wide-range pluripotency of ES cells both in vitro and in vivo. ${ }^{1-4}$ These works have opened up novel therapeutic potentials for a variety of complex disorders. However, most of these studies have focused on two-dimensional (2D) cultures of ES cells under a variety of conditions in order to form cystic embryoid bodies (EBs) and subsequently induce lineage-specific differentiation. Although successful in creating a variety of tissue-specific cell types, these approaches are inherently ineffi- cient and do not mimic in vivo physicochemical conditions. In contrast, three-dimensional (3D) biomaterial scaffolds could provide a significant increase in ES cell differentiation efficiency by enhancing cell-cell and cell-matrix interactions and subsequent cell signaling. Langer and colleagues have reported culture and differentiation of ES cells in 3D polymer scaffolds leading to the formation of neural, hepatic, and mesenchymal tissues. ${ }^{5,6}$ However, studies involving hematopoietic differentiation of ES cells inside biomimetic, 3D environments have not been reported.

With the advent of modern tissue-engineering concepts and emerging cellular transplantation therapies, stem cell-based therapeutics are increasingly becoming a clin-

Department of Biomedical Engineering, University of Texas at Austin, Austin, TX. 
ical reality. Although ES cells have seen limited use in tissue-engineering applications, marrow-derived and adult mesenchymal stem cells are being evaluated by various groups for growing skeletal tissue structures including muscles, bone, cartilage, adipose tissues, and so on. ${ }^{7-10}$ Use of ES cells could provide new directions in tissue regeneration by creating multiple tissue types and organized organ structures inside a single scaffold (i.e., hybrid tissues). ${ }^{1-13}$ In addition, direct transplantation of stem cells (bone marrow transplants) and stem cell-derived cell lineages has proven successful in a variety of complex disorders. The ultimate clinical applicability of stem cell-based therapeutics and tissue engineering requires a fundamental understanding of their behavior under 3D biomimetic conditions and the development of efficient technologies for high-throughput production of progenitors and therapeutic cells suitable for on-demand transplantation.

Current efforts to generate hematopoietic precursor cells (HPCs) involve isolation from patient (or matched donor) bone marrow. Similarly, dendritic cells (DCs) necessary for ex vivo immunotherapy are obtained from peripheral blood cells or in vitro differentiation of HPCs that have been isolated from bone marrow. These methods are constrained by (1) the difficulties and efficiency of isolation, (2) problems with long-term expansion of HPCs in vitro, and (3) limited availability of donor marrows. ${ }^{14}$ In addition, the time required to expand patientisolated HPCs or DCs can often prove to be too long for the patient's survival. Therefore, efficient, high-throughput generation of HPCs and their derivatives from ES cells in a synthetic environment could potentially provide an alternative, more easily available source for therapeutic applications. ${ }^{14}$

Inside a tissue structure, cells are organized within the complex molecular framework of the extracellular matrix $(\mathrm{ECM})$. In addition to providing tissues with the appropriate 3D architecture, ECM molecules also influence cell migration, proliferation, and differentiation through cell-cell and cell-substrate interactions. ${ }^{15-17}$ However, such a complex biological microenvironment does not exist in traditional 2D tissue culture plates. To mimic this $3 \mathrm{D}$ growth environment in vitro, a variety of 3D biomaterials have been used as substitutes for ECM, providing a physical support matrix and increasing cell-cell and cell-substrate interactions. ${ }^{18,19}$ Thus, bioengineered 3D culture systems have become a promising experimental approach, especially for the proliferation and differentiation of progenitor cells. ${ }^{20-22}$

One such material, the Cytomatrix (Cytomatrix, Woburn, MA), is a highly porous and cell-compatible device made of tantalum. The architectural similarities between the Cytomatrix and bone tissue make it particularly well suited for the growth of hematopoietic stem cells. In one study, Cytomatrix was able to enhance the viability and maintain the multipotency of HPCs in longterm cultures without the need for exogenous cytokines, whereas under the same conditions 2D systems were less capable of supporting HPCs. ${ }^{23,24}$ In addition, this 3D cell matrix has also been used to successfully generate human $\mathrm{T}$ cells from $\mathrm{CD} 34^{+}$progenitors in vitro by coculturing them with murine thymic stromal cells. ${ }^{25}$ The critical cell-cell interaction provided by the 3D architecture resulted in more efficient $\mathrm{T}$ cell production compared with a monolayer culture system.

Another bioreactor that has been successfully applied in tissue engineering is the spinner flask, which has been used to regenerate bone tissue ${ }^{26}$ and to culture cell-polymer constructs for cartilage differentiation. ${ }^{27-29}$ Studies have shown that the potential mitigation of external mass transport limitation in a spinner flask culture made it more beneficial for the proliferation and differentiation of marrow stromal osteoblasts seeded on a porous scaffold than a 2D static culture or rotating wall vessel culture, a microgravity-stimulating bioreactor. ${ }^{30}$ Therefore, the unique combination of 3D matrix scaffold architecture and spinner flask technology should provide highly efficient cell growth and differentiation. This microenvironment could enable us to improve viability, maintain pluripotency, and enhance differentiation of progenitor cells while culturing them at tissue-like high densities.

Our overall objective is to study hematopoietic differentiation of ES cells within a biomimetic culture environment. Specifically, we wanted to determine the effects of 3D matrix scaffold alone as well as combined with spinner flask technology on mouse ES cell hematopoietic differentiation and compare them with the effects of the traditional 2D suspension culture. The outcome of this work will provide fundamental insights concerning the basic biology of ES cell differentiation in near-native microenvironments and help us understand how cell-matrix and cell-cell interactions influence stem cells during organ development. Furthermore, the generalized bioengineering concepts developed herein will eventually help to create other cell lineages used in gene therapy, tissue engineering, and organ regeneration.

\section{MATERIALS AND METHODS}

\section{Maintenance and differentiation of ES cells}

R1 murine embryonic stem cells were generously provided by A. Nagy (Mount Sinai Hospital, Toronto, ON, Canada). STO fibroblast cells were a gift from S. Maika (Animal Research Center, University of Texas at Austin). These STO cells have been stably transfected with a transgene for leukemia inhibitory factor (LIF) and thus produce LIF that is secreted constantly into the culture medium. We followed the experimental strategy described by Fairchild et al. ${ }^{31}$ with minor modifications. 
Briefly, undifferentiated ES cells were expanded on irradiation-inactivated STO cells in complete ES cellmaintaining medium containing 15\% ES-Cult fetal bovine serum (Stem Cell Technologies, Vancouver, BC, Canada), $2 \mathrm{mM}$ glutamine, penicillin $\mathrm{G}(100 \mathrm{U} / \mathrm{mL})$, streptomycin $(10 \mu \mathrm{g} / \mathrm{mL}), 0.1 \mathrm{mM}$ nonessential amino acids, and $5 \times 10^{-5} \mathrm{M}$ 2-mercaptoethanol in Iscove's modified Dulbecco's medium (IMDMi-GIBCO-BRL/Invitrogen, Rockville, MD). Medium was changed every 2 days.

Before differentiation, $\mathrm{R} 1$ cells were predifferentiated for $48 \mathrm{~h}$ by passaging free of feeder cells on gelatinized tissue culture plates in complete ES medium, further supplemented with a $1000-\mathrm{U} / \mathrm{mL}$ concentration of recombinant LIF (Chemicon International, Temecula, CA), followed by LIF withdrawal to encourage EB formation. At this stage, single ES cells were either plated onto bacteriological plastic plates at $4 \times 10^{5}$ cells per 90 -mm plate (2D) or seeded onto Cytomatrix (Cytomatrix, Woburn, MA) at $2 \times 10^{5}$ cells per 9 -mm-diameter unit. Cells were seeded onto the Cytomatrix by dropwise addition of cell suspension to each matrix. The scaffolds were incubated for $4 \mathrm{~h}$ in a humidified incubator to allow for cell attachment, followed either by transfer into six-well plates with $2 \mathrm{~mL}$ of culture medium added to each well (3D-0) or assembly into the SW-spinner system (Cytomatrix) according to the manufacturer's protocol (henceforth referred to as 3D-spinner-0). On day 4 of differentiation, some R1 cells, differentiated in 2D plates, were seeded on the Cytomatrix and continued differentiation either in six-well plates (henceforth referred to as 3D-4) or inside the SW-spinner system (henceforth referred to as 3Dspinner-4) as described above (see Table 1 for a summary of experimental groups). The SW-spinner systems were placed on a Cellgro low-speed magnetic stirrer (Barnstead International, Dubuque, IA). Medium was changed every 2 days for each system. To harvest cells off the Cytomatrix, scaffolds were rinsed with Dul- becco's phosphate-buffered saline (D-PBS) (GIBCOBRL/Invitrogen), followed by incubation with $5 \mathrm{~mL}$ of Accumax (Innovative Cell Technologies, San Diego, CA) in a $37^{\circ} \mathrm{C}$ water bath for $10 \mathrm{~min}$, and vortexed two or three times during incubation. The treated scaffolds were washed with $5 \mathrm{~mL}$ of serum-containing medium and centrifuged at $4^{\circ} \mathrm{C}$ for $10 \mathrm{~min}$ at $1000 \mathrm{rpm}$.

For subsequent myeloid differentiation, EBs generated in two dimensions and in 3D-spinner-4 cultures were harvested on day 14 of differentiation and disrupted into single-cell suspensions by incubating them with $3 \mathrm{~mL}$ of collagenase (Stem Cell Technologies) at $37^{\circ} \mathrm{C}$ for $1 \mathrm{~h}$, followed by passage three times through a 21 -gauge, 1.5 in. needle on a $3-\mathrm{cm}^{3}$ syringe. The cells recovered from each 90-mm plate or two Cytomatrix scaffold units were plated onto tissue culture-grade plastic plates in complete ES medium further supplemented with recombinant murine interleukin 3 (rmIL-3, $1000 \mathrm{U} / \mathrm{mL})$, recombinant murine granulocyte-macrophage colony-stimulating factor (rmGM-CSF, $25 \mathrm{ng} / \mathrm{mL})$, rmIL-4 (10 ng/mL), and recombinant murine stem cell factor ( $\mathrm{rmSCF}, 10 \mu \mathrm{g} / \mathrm{mL}$ ) (R\&D Systems, Minneapolis, MN) to promote myeloid differentiation. Medium was changed every 2 days. All cultures were incubated at $37^{\circ} \mathrm{C}$ in $5 \% \mathrm{CO}_{2}$. To activate differentiated myeloid cells, on day 11 of differentiation the floating cells in each plate were harvested and replated into new plates for 3 days with lipopolysaccharide (LPS, $1 \mu \mathrm{g} / \mathrm{mL}$; Sigma, St. Louis, MO) added during the final $24 \mathrm{~h}$ of culture.

\section{Flow cytometry}

Flow cytometry was performed as previously described by Dang et al. ${ }^{32}$ with some modifications. During EB formation, single cells dissociated from EBs were washed with ice-cold Hanks' buffered salt solution (GIBCO-BRL/Invitrogen) containing 2\% bovine serum albumin (BSA; Sigma) (HB buffer) and resuspended at

Table 1. Summary of Various Culture Conditions

\begin{tabular}{ll}
\hline Culture & \multicolumn{1}{c}{ Description } \\
\hline 2D & ES cells were differentiated on tissue culture plates \\
3D-0 & ES cells were seeded directly on Cytomatrix and cultured on six-well \\
plates from day 0 of differentiation & ES cells that have been differentiated on the tissue culture plates plastic \\
3D-4 & were seeded directly on Cytomatrix on day 4 of differentiation followed by \\
& continued differentiation on six-well plates \\
3D-spinner-0 & ES cells were seeded on Cytomatrix and cultured inside the SW-spinner \\
3D-spinner-4 & system from day 0 of differentiation \\
& ES cells that have been differentiated on the tissue culture plates were \\
& seeded on Cytomatrix on day 4 of differentiation followed by \\
& continued differentiation in the SW-spinner system
\end{tabular}


$10^{6}$ cells $/ 100 \mu \mathrm{L}$. Nonspecific binding was blocked by incubating the cells with anti-mouse CD16/CD32 monoclonal antibody ( $1 \mu \mathrm{g} / 100 \mu \mathrm{L}$; PharMingen, Mississauga, $\mathrm{ON}$, Canada) at $4^{\circ} \mathrm{C}$ for $10 \mathrm{~min}$. Test cells were then incubated at $4^{\circ} \mathrm{C}$ for 60 min with a $1-\mu \mathrm{g} / 100 \mu \mathrm{L}$ concentration of fluorescein isothiocyanate (FITC)-conjugated anti-mouse CD34, phycoerythrin (PE)-conjugated antimouse Flk-1, FITC-anti-mouse Sca-1, or PE-anti-mouse c-Kit (PharMingen) for single staining and with a 1$\mu \mathrm{g} / 100 \mu \mathrm{L}$ concentration each of FITC-anti-mouse CD34 and PE-anti-mouse Flk-1 or of FITC-anti-mouse Sca-1 and PE-anti-mouse c-Kit for double staining. After myeloid differentiation, cells were washed with $\mathrm{HB}$ buffer and nonspecific binding was blocked as described above, followed by incubation with a $1-\mu \mathrm{g} / 100 \mu \mathrm{L}$ concentration of FITC-anti-mouse CD11c, FITCanti-mouse MHC class II, or PE-anti-mouse CD11b (PharMingen). Cells used as negative controls were incubated at $4^{\circ} \mathrm{C}$ for $60 \mathrm{~min}$ with fluorochrome-conjugated irrelevant isotype control antibodies at $1 \mu \mathrm{g} / 100 \mu \mathrm{L}$ (PharMingen). In flow cytometry, gates were set so that they covered less than $4 \%$ of the cells in negative controls in each assay (not shown in the histograms of Figs. 3 and 6). The percentage of cells covered by the gate in the test sample minus the percentage of cells covered by the gate in the corresponding negative control was used to represent the percentage of positive cells for each assay. The immunophenotypes of cells were analyzed with a FACScalibur flow cytometer (BD Biosciences Immunocytometry Systems, San Jose, CA) and CellQuest software. Each surface phenotype experiment was repeated independently three times ( $n=3$ each time), and the average of all nine assays is presented along with analysis of statistical significance using a Student $t$ test.

\section{Colony-forming cell assay}

Colony-forming cell (CFC) assays were performed as previously described by Dang et al. ${ }^{32}$ Briefly, on day 10 of differentiation, EBs generated in two dimensions and in 3D-spinner-4 cultures were disrupted into single-cell suspensions as described above. EB cells $\left(5 \times 10^{4}\right)$ were plated into $35-\mathrm{mm}$ petri dishes containing $1.1 \mathrm{~mL}$ of $1 \%$ methylcellulose in IMDM (M3434; Stem Cell Technologies) supplemented with $15 \%$ fetal calf serum, rmSCF (50 ng/mL), rmIL-3 (10 ng/mL), rhIL-6 (10 ng/mL), human erythropoietin (hEpo, $3 \mathrm{U} / \mathrm{mL}), 1 \%$ bovine serum albumin, bovine pancreatic insulin $(10 \mu \mathrm{g} / \mathrm{mL})$, and human transferrin (iron saturated; $200 \mu \mathrm{g} / \mathrm{mL}$ ). The CFCs were observed and scored under bright-field microscope 10 days after plating. CFCs were identified as erythroid burst-forming unit (BFU-E), colony-forming unit-granulocyte/macrophage (CFU-GM), CFU-granulocyte (CFUG), CFU-macrophage (CFU-M), and CFU-granulocyteerythrocyte-macrophage-megakaryocyte (CFU-GEMM).

\section{Scanning electron microscopy and bright-field microscopy}

Both EBs and EB-Cytomatrix complexes were washed with ice-cold D-PBS (GIBCO-BRL/Invitrogen) followed by fixation in $2 \%$ glutaraldehyde (Sigma) in $0.1 \mathrm{M}$ sodium cacodylate buffer ( $\mathrm{pH}$ 7.2) (Polysciences, Warrington, PA) for $1 \mathrm{~h}$ under vacuum. They were then rinsed in sodium cacodylate buffer three times over the course of $45 \mathrm{~min}$, dehydrated in ethanol $(25,50,75$, and $100 \%, \mathrm{v} / \mathrm{v})$, critical point dried, mounted on stubs with double-stick carbon tape, and sputter coated with gold-palladium. The samples were examined with a scanning electron microscope (Philips Electron Optics, Eindhoven, The Netherlands) at
A

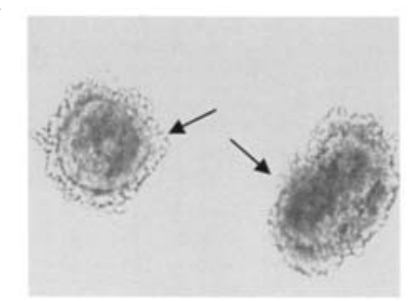

C

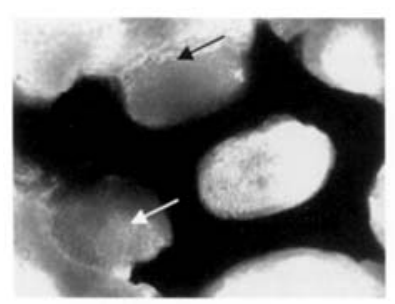

B

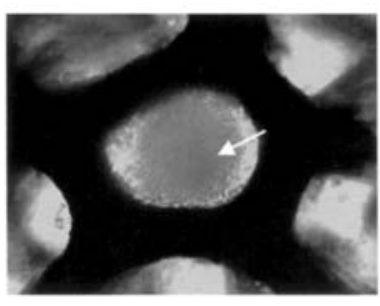

D

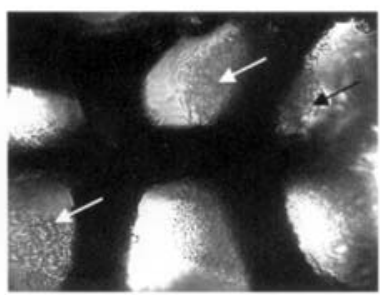

FIG. 1. Efficient formation of EBs in 2D suspension cultures and on Cytomatrix scaffolds. Bright-field micrograph (original magnification, $\times 20$ ) showing EBs (indicated by arrows) formed in 2D suspension cultures (A), inside Cytomatrix pores (B), on the edge of Cytomatrix scaffold walls $(\mathbf{C})$, and embedded within the Cytomatrix network (D). 
an accelerating voltage of $15 \mathrm{kV}$. Bright-field microscopy was performed with an Axiophot polarized photomicroscope (Zeiss, Oberkochen, Germany).

\section{RESULTS}

ES cells were able to differentiate and develop EBs on $3 D$ Cytomatrix scaffolds under both static and dynamic culture conditions

The entire process of ES cell differentiation into DCs in 2D cultures can be divided into two successive phases: generation of HPCs for ES cells and differentiation of DCs from HPCs. ${ }^{31}$ During the first stage, differentiated ES cells form embryoid bodies (EBs), the three-dimensional cell aggregates that contain the precursors of a wide range of cell lineages, including hematopoietic, neural, cardiac myocytie, and pancreatic islet cells. ${ }^{33-36}$ In addition, EBs are also known to provide an effective microenvironment supporting the early stage of hematopoiesis. ${ }^{31}$ Therefore, the efficiency of EB formation and HPC generation directly affects the subsequent frequency of myeloid cell production. During the second stage, HPCs generated in EBs further differentiate into myeloid cells in the presence of specific cytokines.

To our knowledge, differentiation of ES cells on a tantalum-based scaffold matrix has not been reported. Therefore, the first constraint we investigated was whether ES cells could differentiate and form EBs on the Cytomatrix under both static and dynamic culture conditions. Dang et al. $^{32}$ demonstrated that hematopoietic development is sensitive to the timing of EB attachment. EBs attached to a gelatinized surface before day 4 of EB formation resulted in the impairment of hematopoietic mesoderm for- mation and dramatic reduction in hematopoietic cell frequency. To test whether attachment of EBs on the Cytomatrix is also time sensitive, we seeded ES cells on the Cytomatrix on both day 0 and day 4 of differentiation. Each culture was subsequently differentiated either in six-well plates (3D-0 and 3D-4) or inside spinner flasks (3D-spinner-0 and 3D-spinner-4).

In $3 \mathrm{D}$ cultures, EBs formed inside the Cytomatrix under all four conditions, with size and shape similar to those formed under 2D conditions (Fig. 1). In addition, EBs formed in both static and dynamic 3D cultures shared similarities in terms of EB-Cytomatrix interactions: they were either inside the Cytomatrix pores, with attachment occurring in all directions (Fig. 1B), or some of the EBs were attached to the Cytomatrix (Fig. 1C and D). However, despite the morphological similarities, the number of EBs generated in different 3D systems varied: about 20 to 30\% more EBs were observed in 3D-4 and 3D-spinner-4 systems than in their corresponding counterparts, 3D-0 and 3D-spinner-0 systems (data not shown). This is probably because individual ES cells were seeded at an undifferentiated stage in $3 \mathrm{D}-0$ and $3 \mathrm{D}$-spinner-0 cultures, which potentially reduced the rate of ES cell aggregation and EB formation. In contrast, in 3D-4 and 3D-spinner-4 cultures, small EB-like aggregates had already formed during the 4 days of differentiation before seeding on the Cytomatrix.

\section{EBs generated in $2 D$ and $3 D$ cultures possess significantly different biological properties}

Although EBs developed in 2D and 3D cultures were similar in size and shape, they demonstrated significantly different properties. EBs formed in 2D cultures were suspended in the culture medium whereas those generated
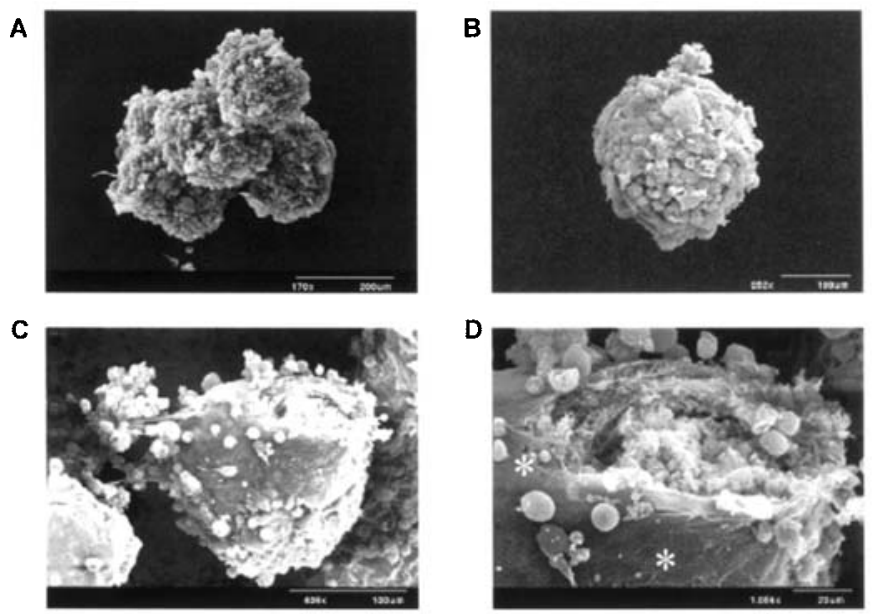

FIG. 2. Properties of EBs generated in 2D suspension cultures and on Cytomatrix scaffolds. Scanning electron micrograph showing that ES cells differentiated in 2D suspension cultures tend to form aggregates of EBs (A) instead of isolated EBs (B). ES cells differentiated on Cytomatrix scaffolds preferentially form single EBs, covered by a membrane-like structure (C and $\mathbf{D}$, indicated by asterisks). 
in $3 \mathrm{D}$ cultures integrated into the matrix scaffolds (Fig. 1). In addition, scanning electron microscopy (SEM) demonstrated that EBs formed in 3D cultures were associated with a membrane-like structure (Fig. 2C and D), which not only covered most of the EB cells (Fig. 2D) but also extended toward the Cytomatrix and formed physical connections between EBs and the Cytomatrix scaffolds (Fig. 2C). However, such a distinctive membrane-like structure was absent on EBs generated in 2D cultures (Fig. 2A and B). On the basis of the morphological properties, we hypothesize that this structure is composed of ECM proteins, which might play an im- portant role in cell-cell and cell scaffold interactions, eventually promoting signal transduction for efficient hematopoietic differentiation.

In addition, EBs generated in 2D suspension cultures frequently formed aggregates and eventually became large EBs (Fig. 2A), which has been proposed to be the main reason behind low ES cell differentiation efficiency. ${ }^{32}$ However, such EB aggregation was rarely observed in our $3 \mathrm{D}$ cultures. We suggest that this was because the attachment of individual EBs on the Cytomatrix scaffold prevented them from floating in the medium or adhering to each other. Thus, our image data suggest that

A

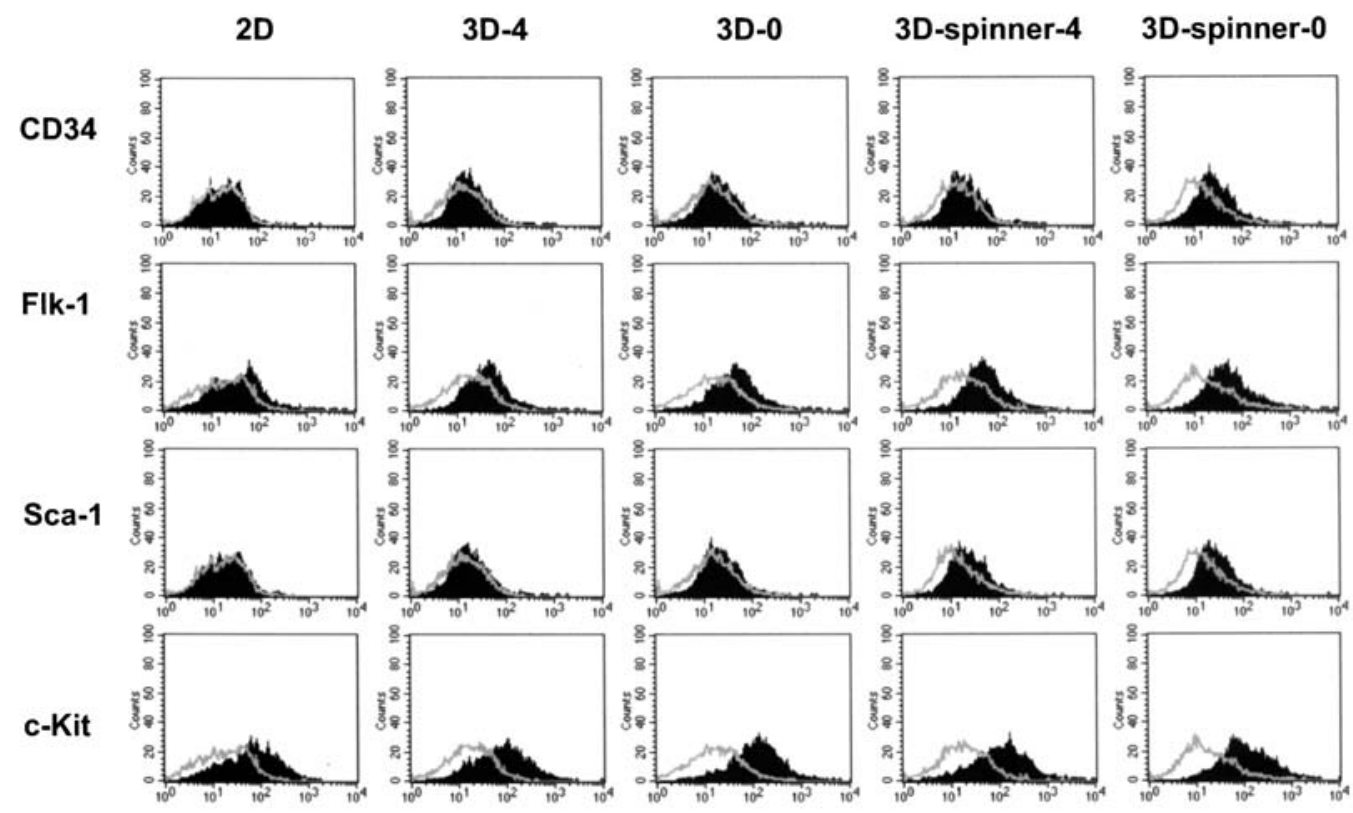

B

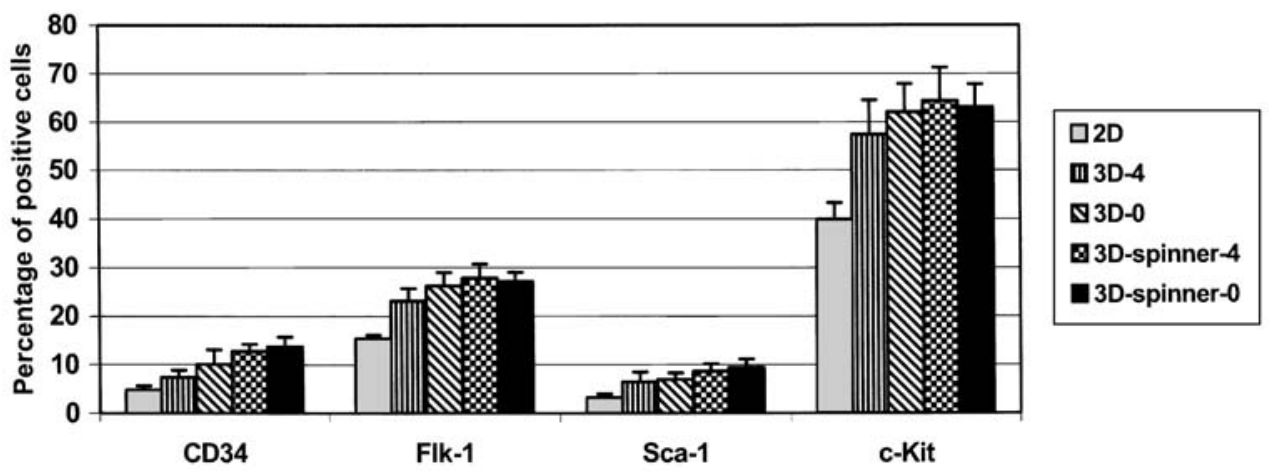

FIG. 3. Expression of hematopoietic cell surface markers on ES cell-derived HPCs generated in 2D and 3D cultures. Flow cytometry analysis (histogram plots) shows the surface immunophenotype of ES cell-derived HPCs in each culture system (A). Solid histograms, levels of expression of the indicated markers; open histograms, background staining with irrelevant speciesand isotype-matched monoclonal antibodies. HPC surface marker expression levels of ES cell-derived HPCs in each system ( $n$ $=9$ from three independent experiments) are compared $(\mathbf{B})$. 


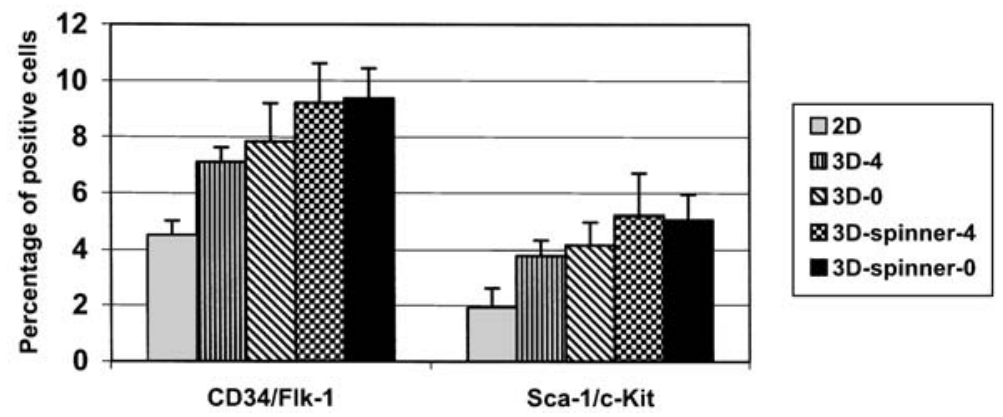

FIG. 4. Dual expression profiles of hematopoietic cell surface markers on ES cell-derived HPCs generated in 2D and 3D systems: comparison of flow cytometry analyses. The percentage of cells positive for indicated dual antibody staining is shown $(n$ $=9$ from three independent experiments).

differentiation of ES cells on the Cytomatrix scaffold not only promoted cell-cell and cell-scaffold interactions but also prevented EB aggregation, both of which should lead to enhanced ES cell differentiation efficiency.

\section{ES cells differentiated in $3 D$ systems demonstrated higher hematopoietic differentiation efficiency}

To evaluate and compare the hematopoietic differentiation potential of ES cells cultured under various 2D and 3D conditions, EBs generated in each system were isolated, disrupted into single-cell suspensions, and analyzed by flow cytometry. Generation of HPCs was evaluated by analyzing several HPC surface markers: CD34, Flk-1, ${ }^{32}$ Sca-1, and c-Kit ${ }^{37-39}$ (Fig. 3).

All 3D cultures showed a significantly higher frequency of HPC generation than their 2D counterparts. Among them, 3D-spinner-0 and 3D-spinner-4 cultures demonstrated the greatest beneficial effects on ES cell hematopoietic differentiation. The expression frequencies of HPC cell surface markers on cells cultured in 3Dspinner cultures were about 1.6 to 2.9 times those of cells differentiated in traditional 2D plates, whereas they were about 1.4 to 2.2 times those of cells cultured under static 3D conditions. Further insight concerning the impact of different culture systems on hematopoietic differentiation potential came from a comparison of cell populations that were positive for dual staining for HPC surface markers. Figure 4 shows that, consistent with the single staining results, compared with $2 \mathrm{D}$ cultures the number of cells that coexpressed CD34 and Flk-1 or Sca-1 and c-Kit was about 2- to 2.7-fold higher in 3D-spinner systems and about 1.6- to 2.2-fold higher in static 3D systems.

All experiments were repeated three different times. Each time a sample size of 3 was used for each culture condition and each surface marker. An ANOVA of the data showed that percentages of cells positive for a specific surface marker (in either single or dual staining) are consistent ( $p>0.05$ for ANOVA) between the three different experiments, indicating that the differentiation process is robust and reproducible (data not shown). Hence for all data analysis processes (bar graphs and $t$ test) a sample size of nine has been used to calculate means and standard deviations.

Student $t$ test analysis of the flow cytometry results (summarized in Table 2) showed that the differences in HPC generation efficiency between 2D suspension cultures and all four 3D cultures were statistically significant $(p<0.05)$. However, no significant difference was observed between the two static 3D cultures, 3D-0 and 3D-4 $(p>0.05)$, or between the two dynamic 3D cultures, 3D-spinner-0 and 3D-spinner-4 $(p>0.05)$, suggesting that the time of EB seeding played a less significant role in hematopoiesis in 3D scaffolds. Interestingly, whereas 3D-0 did not show a highly significant differ-

Table 2. $\quad T$ Test Analysis of Flow Cytometry Data from Various Culture Systems: $P$ Values ${ }^{\mathrm{a}}$

\begin{tabular}{lcccccc}
\hline & CD34 & Sca-1 & Flk-1 & c-Kit & CD34/Flk-1 & Sca-1/c-Kit \\
\hline 2D versus each 3D condition & $<\mathbf{0 . 0 5}$ & $<\mathbf{0 . 0 5}$ & $<\mathbf{0 . 0 5}$ & $<\mathbf{0 . 0 5}$ & $<\mathbf{0 . 0 5}$ & $<\mathbf{0 . 0 5}$ \\
3D-0 versus 3D-4 & $>0.05$ & $>0.05$ & $>0.05$ & $>0.05$ & $>0.05$ & $>0.05$ \\
3D-spinner-0 versus 3D-spinner-4 & $>0.05$ & $>0.05$ & $>0.05$ & $>0.05$ & $>0.05$ & $>0.05$ \\
3D-spinner versus 3D-spinner-0 & 0.096 & $\mathbf{0 . 0 5}$ & $>0.05$ & $>0.05$ & $\mathbf{0 . 0 5 3}$ & $<\mathbf{0 . 0 5}$ \\
3D-4 versus 3D-spinner-4 & $<\mathbf{0 . 0 5}$ & 0.093 & $<\mathbf{0 . 0 5}$ & $>0.05$ & $<\mathbf{0 . 0 5}$ & $<\mathbf{0 . 0 5}$ \\
\hline
\end{tabular}

aPresented are $p$ values for specific cell surface markers. $p<0.05$ indicated in boldface. 
ence from the two dynamic 3D cultures $(p>0.05)$, 3D4 was significantly different from those two cultures in most stainings.

In addition to comparing the frequency of HPC surface marker-positive cells in each culture, we also identified the generation efficiency of functional hematopoietic progenitors through colony-forming cell (CFC) assays. Because of the high efficiency of both EB formation and HPC generation, we chose 3D-spinner-4 as our model system to represent 3D cultures in the CFC assay. EBs derived from 2D and 3D-spinner-4 cultures were disrupted and replated on methylcellulose medium supplemented with cytokines that are necessary for hematopoietic colony formation. All colony types, erythroid burst-forming unit (BFU-E), colony-forming unit-granulocyte (CFU-G), CFU-macrophage (CFU-M), CFU-granulocyte/macrophage (CFU-GM), and CFU-granulocyteerythrocyte-macrophage-megakaryocyte (CFU-GEMM) - were observed in both 2D and 3D-spinner-4-derived cultures. However, the frequency of CFCs in 3D-spinner-4-derived cultures were about 1.8-fold relative to its 2D counterpart $(p<0.05)$ (Fig. 5). Taken together, these data suggest that differentiation of ES cells on the porous 3D biomimetic structures greatly increases their hematopoietic differentiation efficiency. Moreover, rotational culture using spinner flask technology also plays a significant role in promoting ES cell differentiation.

\section{HPCs generated in $3 D$ cultures demonstrated higher differentiation efficiency into myeloid cells possessing DC-like characteristics}

In addition to flow cytometry and CFC assays, the hematopoietic differentiation potential of HPCs generated in 2D and 3D systems was also examined through their ability to further differentiate into the myeloid lineage, specifically dendritic cells. Again, we chose the $3 \mathrm{D}$-spinner-4 system as our model to represent 3D cul- tures in DC differentiation. After 14 days of ES cell differentiation, EBs developed in both 2D and 3D-spinner-4 cultures were harvested and subsequently cultured on $2 \mathrm{D}$ tissue culture plates $(2 \mathrm{D} / 2 \mathrm{D}$ and $3 \mathrm{D}$-spinner-4/2D) in ES cell medium supplemented with murine granulocyte/macrophage colony-stimulating factor (GM-CSF), IL-3, IL-4, and SCF to encourage DC differentiation. In both cultures, cells with an appearance suggestive of DCs appeared within 5 days and subsequently expanded.

To obtain mature DCs, LPS $(1 \mu \mathrm{g} / \mathrm{mL})$ was added to stimulate cell activation. Generation of DC-like cells was validated through both cytometry analysis (Fig. 6) and morphological examination (Fig. 7). Flow cytometry was performed to detect the expression of DC-type surface markers: CD11b, MHC class II, ${ }^{40-43}$ and DC-specific marker CD11c. ${ }^{4-49}$ The examined surface marker expression frequency of the cells derived from 3D-spinner$4 / 2 \mathrm{D}$ was about 2 -fold that derived from 2D/2D culture (Fig. 6), which was consistent with our previous results of HPC generation.

The ontogeny of cells possessing DC-like properties was further demonstrated by their unique morphological characters. Cells differentiated in both 2D/2D and 3Dspinner-4/2D cultures demonstrated the typical morphology of mature dendritic cells, including a highly dendritic appearance and prominent veils of cytoplasm (Fig. 7C and D). Although lightly adherent DC-like aggregates were generated in both cultures, more DC-like clusters as well as more mature dendritic morphology were observed in the $3 \mathrm{D}$-spinner-4/2D system than in $2 \mathrm{D} / 2 \mathrm{D}$ culture (Fig. 7A and B). These results suggest that HPCs generated in the $3 \mathrm{D}$ system possess the ability to further differentiate into a specific hematopoietic lineage. Both cell surface marker expression and morphological characteristics indicate that DC-like cells were produced from ES cell-derived HPCs. However, functional analysis needs to be performed before we can confirm that they are functional DCs.

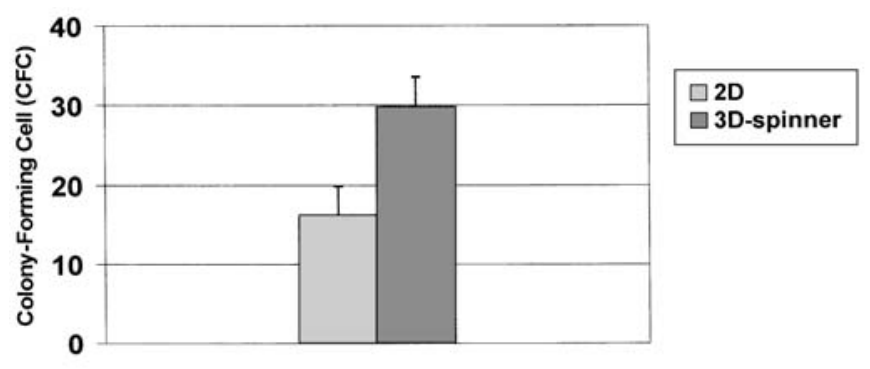

FIG. 5. Colony-forming cell (CFC) assays of ES cell-derived HPCs generated in 2D and 3D-spinner cultures. The number of CFCs was defined as the total number of erythroid burst-forming unit (BFU-E), colony-forming unit-granulocyte (CFU-G), CFUmacrophage (CFU-M), CFU-granulocyte/macrophage (CFU-GM), and CFU-granulocyte-erythrocyte-macrophage-megakaryocyte (CFU-GEMM) $(n=6)$. 
A
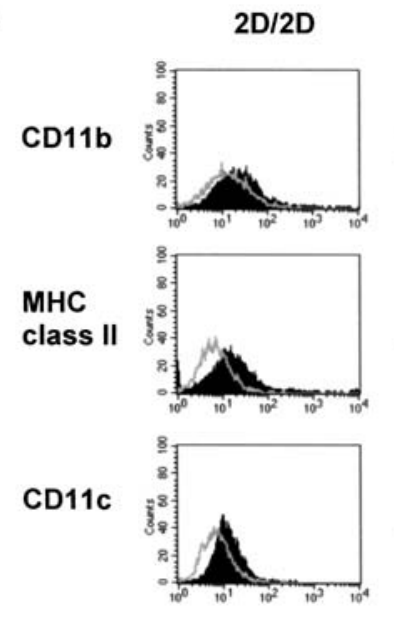

3D-spinner-4/2D
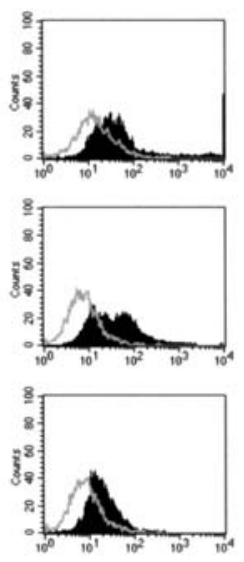

B

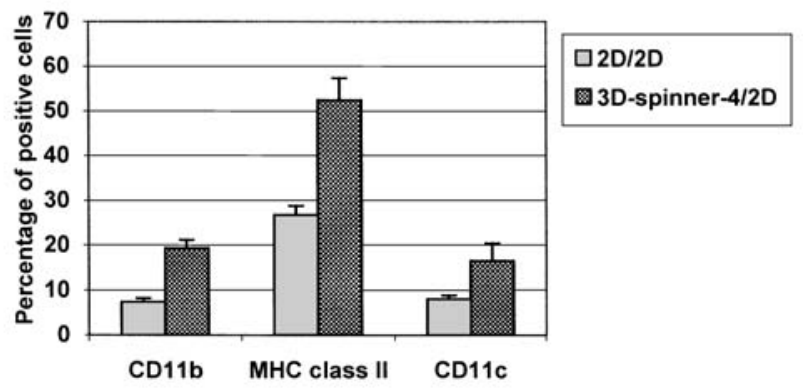

FIG. 6. Expression of dendritic cell surface markers on HPC-derived DC-like myeloid cells. Flow cytometry analysis (histogram plots) showing surface immunophenotype of DC-like myeloid cells differentiated from ES cell-derived HPCs (A). Solid histograms, levels of expression of the indicated markers; open histograms, background staining with irrelevant species- and isotype-matched monoclonal antibodies. Comparison of DC surface marker expression levels on DC-like myeloid cells $(n=3)(\mathbf{B})$.

2D/2D

A

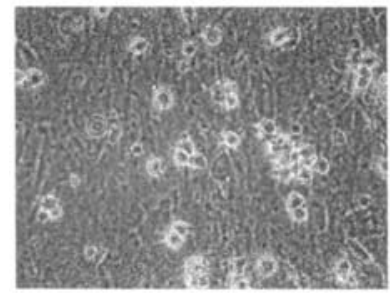

C

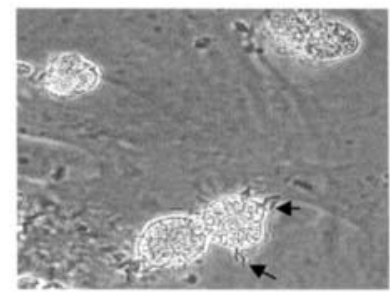

3D-spinner-4/2D
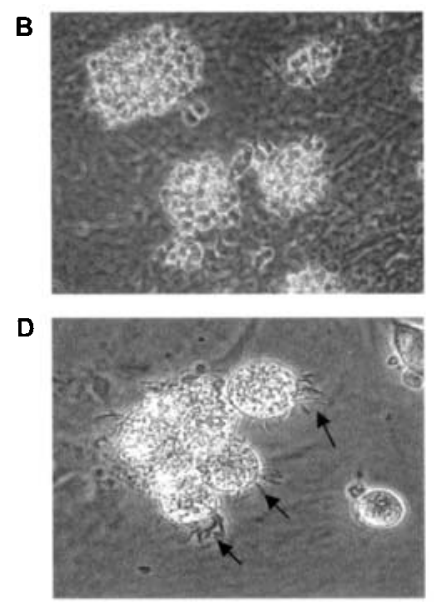

FIG. 7. Activation of HPC-derived DC-like myeloid cells. Phase-contrast micrographs showing that more HPC-derived DClike myeloid cells were generated in the 3D-spinner-4/2D system $(\mathbf{B})$ than in the 2D/2D culture system (A). High-magnification micrographs showing that HPC-derived myeloid cells generated in both systems possess the morphological phenotypes of mature DCs $(\mathbf{C}$ and $\mathbf{D})$. Arrows indicate dendrites on the cell surface. 


\section{DISCUSSION}

This study demonstrates that differentiation of murine ES cells within a biomimetic microenvironment composed of highly porous tantalum-based scaffolds and a spinner flask bioreactor can achieve higher hematopoietic differentiation efficiency than traditional 2D suspension cultures. EBs generated in 2D cultures suspended in medium tend to form aggregates, whereas EBs developed in 3D cultures were integrated into the Cytomatrix scaffolds and rarely aggregated. EBs formed in 3D cultures were covered by a membrane structure reminiscent of the ECM, whereas this structure was absent in EBs generated in the 2D cultures. Most importantly, EBs developed in 3D systems demonstrated a higher efficiency of HPC generation, which was further confirmed by higher frequencies of both hematopoietic colony formation and DC-like myeloid cell production.

Although hematopoietic differentiation of ES cells was more efficient in 3D systems than in 2D suspension cultures, the precise beneficial effects provided by the 3D systems are unknown. The enhanced cell-cell and cell-matrix interactions leading to improved cell signaling in 3D culture may play an important role. Inside the body, hematopoietic stem cells (HSCs) do not grow as independent units. Rather, they are surrounded in all dimensions by the bone marrow niches, which are defined by cell-cell interactions, cell-ECM interactions, and exposure to a variety of soluble factors. ${ }^{50,51}$ These niches play critical roles in regulating stem cell self-renewal, proliferation, survival, differentiation, and mobilization. ${ }^{52}$ For instance, adhesion of HSCs and progenitor cells to the bone marrow ECM prevents apoptosis, which would lead to their long-term survival. ${ }^{50}$ Whereas cell-matrix interactions are greatly impaired on in vitro tissue culture plates, where cells are forced to adjust to an artificially flat and rigid surface, 3D matrices provide a closer approximation to in vivo environment. We suggest that culturing ES cells on 3D Cytomatrix scaffolds would enhance ECM production, which in turn would increase cell-cell and cell-Cytomatrix scaffold interactions and eventually promote the signal transduction that is required for hematopoietic differentiation.

Our data comparing static and dynamic 3D cultures demonstrated that spinner flask technology might also contribute to the higher hematopoietic differentiation efficiency of ES cells seeded on Cytomatrix scaffolds. It has been demonstrated that the mitigation of nutrient transport limitation from external to 3D cell-polymer constructs in spinner flasks increased the proliferation and differentiation of marrow stromal cells seeded on 3D scaffolds. The convective forces generated by the rotation of the magnetic stirrer in the spinner flask enhance nutrient and water transport, thereby decreasing their con- centration gradients on the scaffold surface and the cells seeded inside. ${ }^{30}$ Taken together, the combination of $3 \mathrm{D}$ Cytomatrix scaffold and spinner flask provides a bone marrow-like microenvironment inside a dynamic culture system. Our data suggest that such a culture system may be beneficial in improving HPC expansion, maintaining their viability and preserving their unique biological properties once they are differentiated from ES cells. To elucidate the impacts of different culture conditions on gene expression regulation during ES cell differentiation and to discover the mechanism by which 3D systems enhance ES cell hematopoietic differentiation, DNA microarray analysis is currently being undertaken with RNAs purified from ES cells differentiated in both static and dynamic 3D systems and 2D suspension cultures.

During the stage of DC differentiation, in addition to 2D culture, we also attempted to generate DCs from HPCs in 3D-spinner system (data not shown). However, this system failed to show any conclusive effect on DC differentiation because of the weak attachment of DCs to the Cytomatrix scaffolds. In future, culturing Cytomatrix/ES cell-derived HPCs in a static 3D culture instead of a dynamic spinner flask could provide further insights concerning DC differentiation.

Highly efficient differentiation of ES cells into various hematopoietic progenitor cells under xenogeneic conditions is receiving increasing interest. As a potentially unlimited cell source, ES cell-derived HPCs could be utilized in the field of gene therapy, stem cell transplantation, and transfusion medicine. The production of these clinically important cells relies primarily on the differentiation efficiency of ES cells cultured in vitro. Our study demonstrates that compared with the traditional 2D culture system, the combination of 3D scaffold architecture and spinner flask technology represents a more efficient culture environment for hematopoietic differentiation of ES cells. The 3D culture systems we propose here could also be used to generate viable DCs from ES cells in a more efficient manner, leading to improved DCbased vaccination strategies. Furthermore, these results could provide important insights into studies involving ES cell differentiation into other lineages.

\section{ACKNOWLEDGMENTS}

The authors acknowledge Dr. Andras Nagy (Mount Sinai Hospital, Ontario, Canada) for generously providing R1 murine embryonic stem cells, and Shanna D. Maika (Animal Research Center of the University of Texas at Austin) for kindly giving us STO cells. We also thank John M. Mendenhall (Institute of Cell and Molecular Biology at the University of Texas at Austin) for helping us with confocal microscopy and SEM. 


\section{REFERENCES}

1. Burdon, T., Smith, A., and Savatier, P. Signalling, cell cycle and pluripotency in embryonic stem cells. Trends Cell Biol. 12, 432, 2002.

2. Amit, M., Carpenter, M.K., Inokuma, M.S., Chiu, C.P., Harris, C.P., Waknitz, M.A., et al. Clonally derived human embryonic stem cell lines maintain pluripotency and proliferative potential for prolonged periods of culture. Dev. Biol. 227, 271, 2000.

3. Bremer S., and Vogel, R. Pluripotent stem cells of the mouse as a potential in vitro model for mammalian germ cells: Sister chromatid exchanges induced by MMC and ENU in undifferentiated cell lines compared to differentiated cell lines. Mutat. Res. 444, 97, 1999.

4. Okano, A., Suzuki, C., Takatsuki, F., Akiyama, Y., Koike, K., Ozawa, K., et al. In vitro expansion of the murine pluripotent hemopoietic stem cell population in response to interleukin 3 and interleukin 6: Application to bone marrow transplantation. Transplantation 48, 495, 1989.

5. Levenberg, S., Huang, N.F., Lavik, E., Rogers, A.B., Itskovitz-Eldor, J., and Langer, R. Differentiation of human embryonic stem cells on three-dimensional polymer scaffolds. Proc. Natl. Acad. Sci. U.S.A. 100, 12741, 2003.

6. Levenberg, S., Golub, J.S., Amit, M., Itskovitz-Eldor, J., and Langer, R. Endothelial cells derived from human embryonic stem cells. Proc. Natl. Acad. Sci. U.S.A. 99, 4391, 2002.

7. Krause, D.S. Plasticity of marrow-derived stem cells. Gene Ther. 9, 754, 2002.

8. Peng, H., and Huard, J. Stem cells in the treatment of muscle and connective tissue diseases. Curr. Opin. Pharmacol. 3, 329, 2003.

9. Rogers, J.J., Young, H.E., Adkison, L.R., Lucas, P.A., and Black, A.C., Jr. Differentiation factors induce expression of muscle, fat, cartilage, and bone in a clone of mouse pluripotent mesenchymal stem cells. Am. Surg. 61, 231, 1995.

10. Bruder, S.P., Jaiswal, N., Ricalton, N.S., Mosca, J.D., Kraus, K.H., and Kadiyala, S. Mesenchymal stem cells in osteobiology and applied bone regeneration. Clin. Orthop. 355(Suppl.), S247, 1998.

11. Ho AD, and Punzel M. Hematopoietic stem cells: Can old cells learn new tricks? J. Leukoc. Biol. 73, 547, 2003.

12. Lin, H., Lei, J., Wininger, D., Nguyen, M.T., Khanna, R., Hartmann, C., et al. Multilineage potential of homozygous stem cells derived from metaphase II oocytes. Stem Cells 21, 152, 2003.

13. Ying, Q.L., Nichols, J., Evans, E.P., and Smith, A.G. Changing potency by spontaneous fusion. Nature 416, 545, 2002.

14. Daley, G.Q. From embryos to embryoid bodies: Generating blood from embryonic stem cells. Ann. N.Y. Acad. Sci. 996, 122, 2003.

15. Guan, J.L., and Chen, H.C. Signal transduction in cell-matrix interactions. Int. Rev. Cytol. 168, 81, 1996.

16. Boudreau, N.J., and Jones, P.L. Extracellular matrix and integrin signalling: The shape of things to come. Biochem. J. 339, 481, 1999.
17. Geiger, B., Bershadsky, A., Pankov, R., and Yamada, K.M. Transmembrane crosstalk between the extracellular matrix-cytoskeleton crosstalk. Nat. Rev. Mol. Cell. Biol. 2, 793, 2001.

18. Martin, I., Padera, R.F., Vunjak-Novakovic, G., and Freed, L.E. In vitro differentiation of chick embryo bone marrow stromal cells into cartilaginous and bone-like tissues. J. Orthop. Res. 16, 181, 1998.

19. Tan, W., Krishnaraj, R., and Desai, T.A. Evaluation of nanostructured composite collagen-chitosan matrices for tissue engineering. Tissue Eng. 7, 203, 2001.

20. Solchaga, L.A., Johnstone, B., Yoo, J.U., Goldberg, V.M., and Caplan, A.I. High variability in rabbit bone marrowderived mesenchymal cell preparations. Cell Transplant. 8, 511, 1999.

21. Martin, I., Muraglia, A., Campanile, G., Cancedda, R., and Quarto, R. Fibroblast growth factor-2 supports ex vivo expansion and maintenance of osteogenic precursors from human bone marrow. Endocrinology 138, 4456, 1997.

22. Ripamonti, U., Ma, S., Cunningham, N.S., Yeates, L., and Reddi, A.H. Initiation of bone regeneration in adult baboons by osteogenin, a bone morphogenetic protein. Matrix 12, 369, 1992.

23. Bagley, J., Rosenzweig, M., Marks, D.F., and Pykett, M.J. Extended culture of multipotent hematopoietic progenitors without cytokine augmentation in a novel three-dimensional device. Exp. Hematol. 27, 496, 1999.

24. Baelde, H.J., Cleton-Jansen, A.M., van Beerendonk, H., Namba, M., Bovee, J.V., and Hogendoorn, P.C. High quality RNA isolation from tumours with low cellularity and high extracellular matrix component for cDNA microarrays: Application to chondrosarcoma. J. Clin. Pathol. 54, 778, 2001.

25. Poznansky, M.C., Evans, R.H., Foxall, R.B., Olszak, I.T., Piascik, A.H., Hartman, K.E., et al. Efficient generation of human $\mathrm{T}$ cells from a tissue-engineered thymic organoid. Nat. Biotechnol. 18, 729, 2000.

26. Goldstein, A.S., Juarez, T.M., Helmke, C.D., Gustin, M.C., and Mikos, A.G. Effect of convection on osteoblastic cell growth and function in biodegradable polymer foam scaffolds. Biomaterials 22, 1279, 2001.

27. Gooch, K.J., Kwon, J.H., Blunk, T., Langer, R., Freed, L.E., and Vunjak-Novakovic, G. Effects of mixing intensity on tissue-engineered cartilage. Biotechnol. Bioeng. 72, 402, 2001.

28. Vunjak-Novakovic, G., Obradovic, B., Martin, I., Bursac, P.M., Langer, R., and Freed, L.E. Dynamic cell seeding of polymer scaffolds for cartilage tissue engineering. Biotechnol. Prog. 14, 193, 1998.

29. Riesle, J., Hollander, A.P., Langer, R., Freed, L.E., and Vunjak-Novakovic, G. Collagen in tissue-engineered cartilage: Types, structure, and crosslinks. J. Cell Biochem. 71, 313, 1998.

30. Sikavitsas, V.I., Bancroft, G.N., and Mikos, A.G. Formation of three-dimensional cell/polymer constructs for bone tissue engineering in a spinner flask and a rotating wall vessel bioreactor. J. Biomed. Mater. Res. 62, 136, 2002.

31. Fairchild, P.J., Brook, F.A., Gardner, R.L., Graca, L., Strong, V., Tone, Y., et al. Directed differentiation of den- 
dritic cells from mouse embryonic stem cells. Curr. Biol. 10, 1515, 2000.

32. Dang, S.M., Kyba, M., Perlingeiro, R., Daley, G.Q., and Zandstra, P.W. Efficiency of embryoid body formation and hematopoietic development from embryonic stem cells in different culture systems. Biotechnol. Bioeng. 78, 442, 2002.

33. Bain, G., Kitchens, D., Yao, M., Huettner, J.E., and Gottlieb, D.I. Embryonic stem cells express neuronal properties in vitro. Dev. Biol. 168, 342, 1995.

34. Palacios, R., Golunski, E., and Samaridis, J. In vitro generation of hematopoietic stem cells from an embryonic stem cell line. Proc. Natl. Acad. Sci. U.S.A. 92, 7530, 1995.

35. Rohwedel, J., Maltsev, V., Bober, E., Arnold, H.H., Hescheler, J., and Wobus, A.M. Muscle cell differentiation of embryonic stem cells reflects myogenesis in vivo: Developmentally regulated expression of myogenic determination genes and functional expression of ionic currents. Dev. Biol. 164, 87, 1994.

36. Soria, B., Roche, E., Berna, G., Leon-Quinto, T., Reig, J.A., and Martin, F. Insulin-secreting cells derived from embryonic stem cells normalize glycemia in streptozotocin-induced diabetic mice. Diabetes 49, 157, 2000.

37. Balsam, L.B., Wagers, A.J., Christensen, J.L., Kofidis, T., Weissman, I.L., and Robbins, R.C. Haematopoietic stem cells adopt mature haematopoietic fates in ischaemic myocardium. Nature 428, 668, 2004.

38. Purton, L.E., Bernstein, I.D., and Collins, S.J. All-trans retinoic acid delays the differentiation of primitive hematopoietic precursors $\left(\mathrm{lin}^{-} \mathrm{c}-\mathrm{kit}^{+} \mathrm{Sca}^{-}{ }^{+}\right.$) while enhancing the terminal maturation of committed granulocyte/monocyte progenitors. Blood 94, 483, 1999.

39. Winkler, I.G., Snapp, K.R., Simmons, P.J., and Levesque, J.P. Adhesion to E-selectin promotes growth inhibition and apoptosis of human and murine hematopoietic progenitor cells independent of PSGL-1. Blood 103, 1685, 2004.

40. Lemos, M.P., Fan, L., Lo, D., and Laufer, T.M. CD8 ${ }^{+}$and $\mathrm{CD}_{11 b^{+}}$dendritic cell-restricted MHC class II controls Th1 CD4 ${ }^{+}$T cell immunity. J. Immunol. 171, 5077, 2003.

41. Pulendran, B., Dillon, S., Joseph, C., Curiel, T., Banchereau, J., and Mohamadzadeh, M. Dendritic cells generated in the presence of GM-CSF plus IL-15 prime potent $\mathrm{CD}^{+} \mathrm{Tc} 1$ responses in vivo. Eur. J. Immunol. 34, 66, 2004.

42. Johansson C., and Wick, M.J. Liver dendritic cells present bacterial antigens and produce cytokines upon Salmonella encounter. J. Immunol. 172, 2496, 2004.

43. Daro, E., Butz, E., Smith, J., Teepe, M., Maliszewski, C.R., and McKenna, H.J. Comparison of the functional proper- ties of murine dendritic cells generated in vivo with Flt3 ligand, GM-CSF and Flt3 ligand plus GM-SCF. Cytokine 17, 119, 2002.

44. Suter, T., Malipiero, U., Otten, L., Ludewig, B., Muelethaler-Mottet, A., Mach, B., et al. Dendritic cells and differential usage of the MHC class II transactivator promoters in the central nervous system in experimental autoimmune encephalitis. Eur. J. Immunol. 30, 794, 2000.

45. Karman, J., Ling, C., Sandor, M., and Fabry, Z. Dendritic cells in the initiation of immune responses against central nervous system-derived antigens. Immunol. Lett. 92, 107, 2004.

46. Sato, M., Iwakabe, K., Ohta, A., Sekimoto, M., Nakui, M., Koda, T., et al. Functional heterogeneity among bone marrow-derived dendritic cells conditioned by $\mathrm{T}_{\mathrm{H}} 1$ - and $\mathrm{T}_{\mathrm{H}} 2$ biasing cytokines for the generation of allogeneic cytotoxic T lymphocytes. Int. Immunol. 12, 335, 2001.

47. Xanthou, G., Tapinos, N.I., Polihronia, M., Nezis, I.P., Margaritis, L.H., and Moutsopoulos, H.M. CD4 cytotoxic and dendritic cells in the immunopathologic lesion of Sjögren's syndrome. Clin. Exp. Immunol. 118, 154, 1999.

48. Man, A.L., Bertelli, E., Regoli, M., Chambers, S.J., and Nicoletti, C. Antigen-specific T cell-mediated apoptosis of dendritic cells is impaired in a mouse model of food allergy. J. Allergy Clin. Immunol. 113, 965, 2004.

49. Chambers, S.J., Bertelli, E., Winterbone, M.S., Regoli, M., Man, A.L., and Nicoletti, C. Adoptive transfer of dendritic cells from allergic mice induces specific immunoglobulin $\mathrm{E}$ antibody in naive recipients in absence of antigen challenge without altering the $\mathrm{T}$ helper $1 / \mathrm{T}$ helper 2 balance. Immunology 112, 72, 2004.

50. Krause, D.S. Regulation of hematopoietic stem cell fate. Oncogene 21, 3262, 2002.

51. Watt, F.M., and Hogan, B.L. Out of Eden: Stem cells and their niches. Science 287, 1427, 2000.

52. Rafii, S., Avecilla, S., Shmelkov, S., Shido, K., Tejada, R., Moore, M.A., et al. Angiogenic factors reconstitute hematopoiesis by recruiting stem cells from bone marrow microenvironment. Ann. N.Y. Acad. Sci. 996, 49, 2003.

Address reprint requests to: Krishnendu Roy, Ph.D. Department of Biomedical Engineering University of Texas at Austin ENS 610, C0800, 1 University Station Austin, TX 78712

E-mail: kroy@mail.utexas.edu 